\title{
The Relevance of Videos as a Practical Tool for Communication and Dissemination in Horizon2020 Thematic Networks
}

\author{
Elena Feo $^{1, * \mathbb{D}}$, Hannes Mareen ${ }^{2} \mathbb{D}$, Sylvia Burssens ${ }^{3}$ and Pieter Spanoghe ${ }^{1}(\mathbb{D}$ \\ 1 Laboratory of Crop Protection Chemistry, Department of Plants and Crops, Ghent University, \\ 9000 Ghent, Belgium; pieter.spanoghe@ugent.be \\ 2 Internet Technology and Data Science Lab-IDLab, imec, Department of Electronics and Information Systems, \\ Ghent University, 9000 Ghent, Belgium; hannes.mareen@ugent.be \\ 3 Research Coordination Office_EU Team, Ghent University, 9000 Ghent, Belgium; sylvia.burssens@ugent.be \\ * Correspondence: elena.feo@ugent.be
}

check for

updates

Citation: Feo, E.; Mareen, H.; Burssens, S.; Spanoghe, P. The Relevance of Videos as a Practical Tool for Communication and Dissemination in Horizon2020 Thematic Networks. Sustainability 2021, 13, 13116. https://doi.org/ $10.3390 /$ su132313116

Academic Editor: Leyla

A. Gamidullaeva

Received: 12 October 2021

Accepted: 24 November 2021

Published: 26 November 2021

Publisher's Note: MDPI stays neutral with regard to jurisdictional claims in published maps and institutional affiliations.

Copyright: (C) 2021 by the authors. Licensee MDPI, Basel, Switzerland. This article is an open access article distributed under the terms and conditions of the Creative Commons Attribution (CC BY) license (https:/ / creativecommons.org/licenses/by/ $4.0 /)$.
Abstract: Thematic networks (TNs) are Horizon2020 projects promoted by the European Innovation Partnership Agricultural Productivity and Sustainability (EIP-AGRI) that collect existing knowledge and best practices on a given theme in agriculture and forestry. Their purpose is to make these available in easily understandable formats for users such as farmers/foresters and advisors. Videos are a powerful tool for communication and dissemination about project outcomes; thus, they are frequently used as part of project activities. Despite the large number of videos that have been produced by TNs, only a few have reached a high number of views. Hence, most have not reached a wide public, decreasing the impact of TNs' outputs. This paper analyzes several characteristics of $\mathrm{TN}$ videos in order to suggest best practices. That is, we investigated whether each characteristic influences the number of views or average percentage watched. Using the analysis, we suggest several best practices. For example, future TNs should show practical content prioritizing farmers as a speaker and translate videos in more than one language. Better involvement of project partners increases the contact with farmers; thus, outcomes are more easily spread, and the chance of being applied increases. To increase the visibility of videos, a user-friendly digital knowledge platform linked to demonstration activities and peer-to-peer exchange will improve the sharing of knowledge. This will further enhance a TN's impact and sustainability in agricultural and forestry innovation in the longer term.

Keywords: agriculture and forestry sustainability; innovation; impact; rural development; agricultural policy; EIP-AGRI; AKIS

\section{Introduction}

Co-creating and sharing existing knowledge and best practices in a given agriculture and forestry theme is the core idea of Horizon2020 thematic networks (TNs). TNs collect practice-oriented scientific knowledge in animal and plant production and cross-sectoral themes such as rural development. This thematic knowledge is close to being put into practice but is not yet sufficiently ready for farmers and foresters to implement. The main aim of TNs is, then, to collect and translate this knowledge into easily understandable material, which provides informative recommendations, guidelines, solutions, and best practices.

These projects are promoted by the European Innovation Partnership Agricultural Productivity and Sustainability (EIP-AGRI). The EIP-AGRI promotes innovation in the agricultural and forestry sector and brings research and practice closer together using the "interactive innovation model". The aim is to foster competitive and sustainable agriculture and forestry sector in order to achieve more but consume less. In general, agricultural and forestry sustainability rests on the principle that meeting the needs of the present generation does not compromise the ability of future generations to meet their own needs. 
To achieve this aim, the EIP-AGRI funds projects such as TNs to co-create knowledge and promote sustainable innovation.

The conceptual framework in which these actors are connected (at a regional, national, or local level) is represented by the agricultural knowledge and innovation system (AKIS) [1]. Following the interactive model, TNs focus on linking different types of actors with innovation in agriculture or forestry. This gives them the possibility to collaborate, share their ideas, and turn existing knowledge and research results into innovative solutions that can more easily be put into practice [2]. This approach is also known as the multi-actor approach (MAA) and represents the actor's joint forces in project activities, from beginning to end [3-5].

To be impactful, TNs translate their results into easily understandable user materials, such as short, informative recommendations and solutions (practice abstracts), leaflets, guidelines, and audio-visual material (e.g., photos, videos, podcasts, and infographics). The knowledge is co-generated (collected) and co-translated by actors engaged inside and outside the TN consortium (Figure 1, step 1). The produced materials are made available beyond the lifespan of the project, to be used by actors that are not directly involved in TN activities. (Figure 1, step 2 and step 3) [5,6].

Step 1
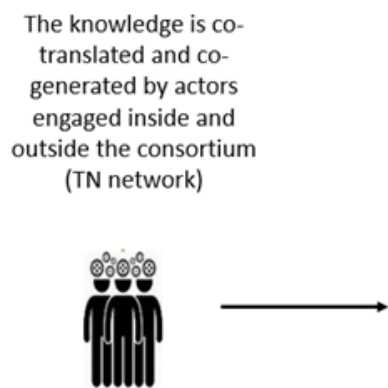

Step 2

The TN collected Knowledge is multiplied to actors that are not directly involved in TN activities (peer to peer exchange)
Step 3

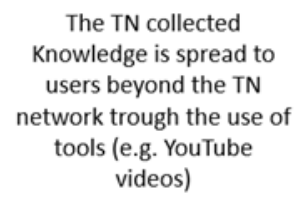

Figure 1. Steps of knowledge generation and exchange. Step 1: co-generation and co-translation. Step 2: the knowledge is multiplied by actors not directly involved in TN activities (e.g., through on-farm demonstration, field visits, peer-to-peer exchange). Step 3: The knowledge is spread to actors beyond the TN. Illustration adapted from EURAKNOS, 2020.

To spread TN results as far as possible and to ensure that the gained knowledge or exploitable foreground can benefit the whole society, a thorough communication and dissemination $(C \& D)$ plan must be structured at the beginning of the project $[7,8]$. This plan allows a TN to achieve a high impact, which is defined as the societal, economic, and environmental effect that EU projects have on society [9]. In other words, achieving high impact means that TNs successfully provide access to meaningful, practice-oriented knowledge to targeted actors. This improves the processes of seeking and implementing information relevant to agriculture and forestry.

In practice, there is often confusion about the difference between communication and dissemination. According to the European Commission [10] and the Horizon Europe Programme [11], communication "is a strategically planned process that aims at promoting project action and its results. It requires strategic and targeted measures for communicating about (1) the action and (2) its results to a multitude of audiences, including the media and the public, and possibly engaging in a two-way exchange", while dissemination is "the public disclosure of the real results by any appropriate means to be used, including by scientific publications in any medium". From the study conducted in the EURAKNOS project, this distinction between communication and dissemination is not always clear between the project members. As such, this may cause a wrong C\&D strategy throughout the life of TNs. Moreover, the C\&D channels strongly depend on the different types of actors, their age, country, and socio-economic backgrounds [6,12]. For example, Irish advisors prefer Twitter over Facebook, and farmers YouTube over Twitter. Videos are becoming 
more frequently used as part of project C\&D activities of TNs. They play an increasingly important role in the agricultural and forestry sector, as they are an excellent way to show the direct experiences of practitioners [13]. During the lifetime of a TN, researchers, advisors, farmers, and other actors produce videos to capture their observations and share new knowledge regarding a specific TN topic. Recently, this has become easier, since video equipment (e.g., smartphones) and editing software are inexpensive and easy to use [14]. Furthermore, farmers often prefer videos over written resources to be informed about a specific topic or approach in their fields [13].

TNs provide a wide range of typology suitable for presentation in the form of videos (e.g., demonstrations, training, tutorials, and events). Usually, TNs upload those materials in their YouTube channel, since the connection rate between farmers and YouTube is high [15]. An additional advantage is that YouTube is easily accessible through cell phones [13]. More specifically, according to the study of Van Mele et al. [16], the percentage of farmers accessing video platforms via their mobile phones in Europe has increased from $30 \%$ in 2016 to $42 \%$ in 2018.

In the H2020 EURAKNOS project, a thorough analysis of the used social media in TNs and of the user engagement was performed. EURAKNOS supported the exchange of existing approaches, methodologies, and tools between TNs. Additionally, the project explored the feasibility of setting up a European agricultural and forestry knowledge and innovation open-source platform that collects outputs and materials produced by TNs. In other words, the digital knowledge will be centralized and made accessible in a sustainable way for the agricultural and forestry innovation community in Europe [17]. This will increase the sharing of TN videos and hence their number of views and the effective implementation of their content. Furthermore, the creation of such a platform aims to improve the sustainability and competitiveness of the agricultural sector. In this way, it may simplify the daily work of agricultural and forestry advisors and farmers (among other actors) [18].

Advisors represent key intermediary actors in the AKIS, as they have the potential to boost innovation. They do this by funneling practice needs into research activities, intermediating in farmer-to-farmer learning processes, and by capturing grass-roots innovative ideas from practice for further development. Their task can vary, depending on the typology of farmers/foresters they are assisting (e.g., arable farm, dairy farm, forest management) [19].

Finally, the European Commission calls on the member states to take advantage of the potential of such platforms and digitization in agriculture. This is necessary to achieve the objectives set by the current and future common agricultural policy (CAP), where digitalization is present in the general objectives, in the specific objectives, in the chapter on knowledge systems in agriculture, and the chapter on agricultural modernization [20].

A large number of videos have been produced by TNs since their launch in 2015. However, only a few of them have reached a high number of views (i.e., more than 1000). Hence, the message they want to convey does not reach a high number of users, which decreases the impact of TN outputs.

In recent years, many studies regarding the importance of using videos for knowledge exchange with farmers were performed both inside and outside Europe [14,21,22]. For example, Van Campenhout et al. (2021) found that, after watching videos related to their practice, maize farmers applied the suggested recommendation in the field [23]. Hence, their production increased. Additionally, Sodagar et al. (2021) confirmed that videos are efficient tools for learning in the forestry context. That is because they help foresters to become acquainted with natural protected environments, preventing environmental violations [24]. Moreover, Karubanga et al. (2019) highlight the efficacy of videos as a complementary tool to other extension approaches in efforts to effectively disseminate relevant information [25].

In general, there are two main limitations to these studies. First, most studies were field surveys that interview a small subset of users on the importance of videos as a learning 
tool. Furthermore, these studies did not consider quantitative metrics of the videos that are impacted by all users, such as the number of views per video on YouTube. Additionally, none of these studies were applied to TN videos. Since an increased number of videos have been produced by TNs in the EU digital knowledge exchange context, their effective efficiency and characteristics have not been studied yet. Furthermore, the final report documents of TNs do not mention YouTube analytics as an indicator of their impact. Hence, the strengths and weaknesses of using videos for TN C\&D are not known. This is where this study aims to fill the gap in order to suggest best practices for future TNs.

The main research question is which characteristics of videos have an objectively measured influence on their impact? More specifically, this study investigates the characteristics of 518 videos produced by 16 completed TNs that ran between 2015 and 2020. The analysis enables us to provide indications on video best practices for future TNs in order to maximize their message efficacy towards their audience.

\section{Materials and Methods}

\subsection{Data Collection}

The analysis of TN videos was performed between May and July 2020, and it consisted of two steps. Firstly, 16 TNs that finished their mandate at the end of 2019 were identified. The other 12 TNs that were still running at the moment of the analysis were not included, due to the fact that not all videos may have been produced and disseminated yet at an early TN stage. The information regarding the year of the analyzed TNs was retrieved in the Community Research and Development Information Service (CORDIS).

As a second step, once the 16 TNs were identified, their webpage was consulted to find if the TNs produced video materials. The collected videos were then analyzed, and their characteristics were evaluated, as well as their total number of views at the time of analysis. Table A1 describes the characteristics of the video that were considered.

Additional to the publicly available number of views of each video, we requested the average percentage viewed of each video, which is only available to the owner of the corresponding YouTube channel. In total, 5 TNs (AFINET, Inno4GRASS, OK NET ARABLE, SMART AKIS, and WINENETWORK) positively responded to our request and enabled us to do this analysis, while the other TNs did not respond or could not find the YouTube login credentials. These TNs collectively produced 90 videos. As such, we could only perform the analysis of more in-depth YouTube analytics for a subset of the data.

All the collected information was retrieved from the YouTube and Vimeo platform, since TNs only used these two platforms to upload their videos. Social media analytics related to the number of subscribers to the YouTube channel was found in the final C\&D plan of the analyzed TNs.

The categorization used to categorize the content type was made based on the guidelines provided by Alföldi et al. [26], where 7 standard typologies of videos in agriculture were defined (Machines demonstration, training videos, tutorial, practice innovation, event and teaser videos, result of research). However, TN activities that can be translated into a video go beyond the range of topics suitable for videos presented in the guideline. Table A2 presents the subdivision used in this study at the relative explanation.

The association of these 16 TNs with each of the topics "Crop farming", "Livestock", and "Forestry" was attributed based on the classification available in the EIP-AGRI database [27] that provided access to TN-related information and field of work. The textual descriptions of TNs were provided as input to the cortical.io web application for the extraction of TN-related keywords. Once the keywords were identified, it was possible to classify the TNs.

\subsection{Analysis}

The created dataset was analyzed using the software for statistical computing and graphics " $R$ ". We created bar charts and box plots to compare the influence of each of the characteristics listed in Table A1. For each TN, the box plot shows the median number 
of views, as well as the lower and upper quartile, smallest and largest non-outlier values connected by the horizontal lines (whiskers), as well as outliers. Additionally, the vertical height of a box represents the number of videos made. Note that the boxplots shown in this paper were made using a logarithmic scale for the number of views. A logarithmic scale was used, because there are few outliers with more than 10,000 views, whereas most videos have fewer than 1000 views. Hence, these videos would be hard to compare on a linear scale.

The box plots helped us visually inspect the influence of each characteristic type on the number of views. In order to make a more quantitative observation on whether certain characteristics influence the number of views, we additionally calculated Kendall's Tau-b rank correlation coefficient for the continuous, ordinal, and selected binary variables. Additionally, we calculated the correlation for selected handcrafted binary variables (e.g., content-type: no presentation vs. presentation, or speaker (researcher vs. farmer)). Kendall's correlation coefficient is a value between -1 and +1 , where -1 indicates a perfect negative correlation, +1 is a perfect positive correlation, and 0 is no correlation whatsoever. Additionally, the corresponding z-score and $p$-value are calculated to verify whether the obtained results are statistically significant. In addition to the number of views, we also analyzed the influence of video characteristics on the average percentage that it was watched (for a subset of the dataset). This was also done using Kendall's correlation coefficient. Finally, we verified if the observations and conclusions we made can also be drawn for each individual TN. If this was not the case, we investigated the videos of those TNs in more depth.

\section{Results and Discussion}

This section presents the results obtained from our study and discusses the related best practices. First, Section 3.1 describes some general statistics from our assembled dataset. Then, the subsequent sections discuss the influence of the characteristics given in Table A1.

\subsection{General TN Video Statistics}

A total of 517 videos were produced by the analyzed TNs. YouTube is confirmed as the most-used platform: all the analyzed TNs stored their videos on YouTube, except AGRIFORVALOR, which used Vimeo. Table 1 reports the number of videos, number of subscribers, and the main target audience for the YouTube channels of the analyzed TNs.

Table 1. Number of videos, number of subscribers, and main target audience of the 16 investigated TNs based on the information available in the final C\&D plan.

\begin{tabular}{cccc}
\hline Thematic Network & Number of Videos & Number of Subscribers & TN Main Target Audience \\
\hline FERTINNOWA & 138 & 47 & $\begin{array}{c}\text { Growers, advisors, policymakers } \\
\text { Farmers, veterinarians, policymakers, } \\
\text { advisors, researchers }\end{array}$ \\
SHEEPNET & 114 & 121 & Farmers, veterinarians, policymakers, \\
advisors, researchers \\
4D4F & 45 & 311 & Farmers, policymakers, advisors, researchers \\
Inno4GRASS & 40 & 122 & Farmers, policymakers, advisors, researchers \\
SKIN & 34 & 49 & Policymakers, advisors \\
AGRISPIN & 30 & 173 & Farmers, policymakers, advisors, researchers \\
HNV-LINK & 28 & 57 & Foresters, policymakers, advisors, researchers \\
AFINET & 20 & 172 & Farmers, policymakers, advisors \\
CERERE & 19 & 65 & Farmers, policymakers, researchers \\
WINETWORK & 18 & 10 & Farmers, policymakers, advisors \\
AGRIFORVALOR & 10 & N/A & Farmers, policymakers, advisors, researchers \\
OK NET ARABLE & 8 & 30 & Farmers, policymakers, advisors, researchers \\
SMART AKIS & 4 & 14 & Farmers, policymakers, advisors, researchers \\
HENNOVATION & 4 & 12 & Farmers, policymakers, advisors \\
EURODAIRY & 4 & 29 & Farmers, policymakers, advisors, NGOs \\
EUFRUIT & 1 & N/A &
\end{tabular}




\subsection{Number of Videos per TN}

Table 1 shows the number of videos produced per TN. We can see that some TNs produced significantly more videos than others. For example, FERTINNOWA produced more than 100 videos, whereas EUFRUIT produced only one. The number of produced videos generally depends on the number and typology of activities that each TN perform. Additionally, it depends on what was agreed in the project proposal. On average, the number of videos produced per TN is 32.3 , the median value is 19.5 , and the standard deviation is 38. This standard deviation is high, because it is distorted by few TNs that produced significantly more videos than the mean (such as FERTINNOWA and SHEEPNET with 138 and 114 videos, respectively).

We observed that having a large number of videos does not necessarily mean that they are watched more often. This is illustrated in Figure 2, which shows boxplots of the number of views for each TN, ordered according to the number of videos in that TN (as in Table 1). The majority of the videos of the 16 TNs have fewer than 1000 views. A good example is provided by the TN FERTINNOWA. It has the largest number of videos, but also the lowest median number of views (12.5). On the contrary, AFINET produced fewer videos, but the median number of views is high (355.5). In general, we observed a moderately negative correlation between the number of videos of a TN and their number of views. That is, Kendall's rank correlation coefficient between the videos' number of views and the number of videos in the corresponding TNs is approximately -0.45 . No correlation is found with the average percentage viewed for the subset of TNs, as the correlation coefficient is approximately 0.16 , and the $p$-value is 0.05 . These results, as well as the correlation results of the other characteristics, are reported in Table 2. In general, it is possible to deduce that more videos certainly does not mean more views; hence, quantity does not guarantee their popularity.

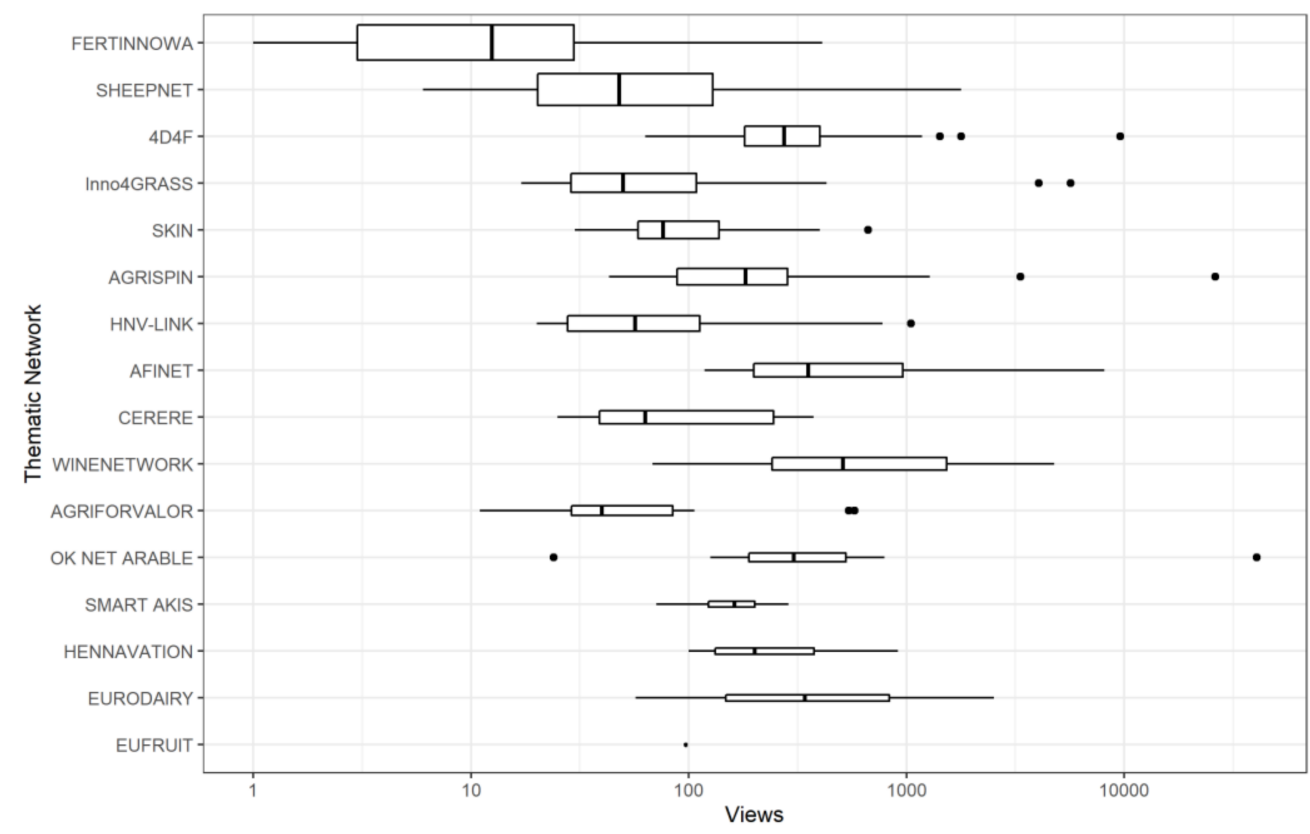

Figure 2. The boxplots of the number of views for all videos of each TN, ranked according to the number of videos that these TNs created. 
Table 2. Correlation results of selected video characteristics. The $p$-value is smaller than 0.001 for all reported values, except when explicitly mentioned otherwise. Additionally, the values are colored in grey when the $p$-value exceeds 0.05 . Note that the correlation with the average percentage viewed is calculated using only a subset of the TNs.

\begin{tabular}{|c|c|c|}
\hline \multirow{2}{*}{ Variable } & \multicolumn{2}{|c|}{ Kendall's Rank Correlation Coefficient } \\
\hline & Views & Average Percentage Viewed \\
\hline Number of videos in TN & $-0.45(z=-14.307)$ & $0.16(z=1.9844, p=0.04721)$ \\
\hline $\begin{array}{l}\text { Release date: Beginning vs. } \\
\text { Middle vs. End }\end{array}$ & $-0.29(z=-8.2594)$ & $0.16(z=1.8194, p=0.06885)$ \\
\hline $\begin{array}{l}\text { Communication vs. } \\
\text { Dissemination }\end{array}$ & $0.15(z=4.1174)$ & $0.05(z=0.55816, p=0.5767)$ \\
\hline $\begin{array}{c}\text { Content Type: No Presentation vs. } \\
\text { Presentation }\end{array}$ & $-0.43(z=-11.786)$ & $-0.26(z=-2.9529, p=0.003148)$ \\
\hline Speaker: Researcher vs. Farmer & $0.24(z=4.2647)$ & $0.27(z=2.3331, p=0.01964)$ \\
\hline Number of subtitles & $0.39(z=11.397)$ & $-0.11(z=-1.3724, p=0.1699)$ \\
\hline $\begin{array}{c}\text { Video length:Less than vs. more } \\
\text { than } 10 \text { mins }\end{array}$ & $-0.32(z=-8.9123)$ & $-0.22(z=-2.501, p=0.01238)$ \\
\hline
\end{tabular}

\subsection{TN Topic}

Regarding the topics or themes, five TNs worked with livestock topics (SHEEPNET, 4D4F, INNO4GRASS, HENNOVATION, and EURODAIRY), ten TNs worked with crop farming topics (FERTINNOWA, SKIN, AGRISPIN, HNV-LINK, CERERE, AGRIFORVALOR, OK-NET ARABLE, SMART AKIS, and EUFRUIT), and only a single TN (AFINET) worked in the forestry field. The median number of views of AFINET, the only TN related to forestry, is high (355.5), whereas TNs that produced videos in livestock and crop-farming topics had lower median numbers of views (87 and 43 , respectively).

\subsection{Release Date and Communication vs. Dissemination}

The majority of videos are made and released at the end of TN's lifespan. These "last-minute" videos result in a smaller median number of views, namely 38 in contrast to 191 and 139 for videos made at the beginning and middle, respectively, of the TN's lifespan. This is confirmed by Kendall's rank correlation coefficient between the videos' number of views and the release period (beginning vs. middle vs. end), which is approximately -0.29 , suggesting a moderate negative correlation. No correlation is found with the average percentage viewed for the subset of TNs, as the correlation coefficient is approximately 0.16 , and the $p$-value is 0.07 . The lower number of views can be explained by the fact that videos published in the end have less time to be viewed. Additionally, and perhaps more importantly, videos produced at the end of a project's lifespan might be promoted less. In case follow-up projects are granted, they may continue the dissemination of the latest videos to enhance their diffusion.

We additionally observed this relation for each individual TN, except for AFINET, for which videos produced at the end generally have more views than those produced in the beginning. It should be noted that AFINET produced most videos at the end. A reason for this could be that videos produced at the end contained multiple subtitles, in contrast to those at the beginning. As discussed in Section 3.7, this is a strong influencing factor.

The majority of the videos ( $98 \%$ ) were related to the dissemination of project results. Communication represented only the remaining $2 \%$. The fact that only $2 \%$ of the videos are related to communication, confirms the outcome of the EURAKNOS investigation on C\&D activities in TNs $[15,28]$. In fact, from that analysis, it emerged that videos are mostly used for the dissemination of the TN's specific results and not for the communication of the project.

The few communication videos all have a large number of views (i.e., more than 1000). Kendall's correlation coefficient between the video's number of views and whether the video is for dissemination or communication purposes is approximately 0.15 . This 
suggests a slight correlation. No correlation is found with the average percentage viewed, though. The fact that the number of views for videos with TN communication purposes is high can be explained by the fact that communication videos mainly aim to target a wider audience, including the media and the general public. Project results are often not involved. Instead, a general overview of the project with a description of the agricultural and forestry challenges is provided. Less technical language is used, so that a non-specialist audience can easily understand the goals and means of the project.

Due to their high popularity yet only being the minority of the produced videos, TNs should consider producing more videos that communicate about their project. This may stimulate the audience's curiosity to further investigate TN results. This should be made clear in the C\&D plan, where the disclosure of such videos needs to be planned thoroughly at the beginning of the project.

Furthermore, social media is a strong vector to stimulate access to project outcomes. Therefore, partners should be more encouraged to use them to promote TN videos, taking advantage of their network outside the TN community. If used strategically, social media is also a vector to grow the community of people around the project. For example, before the end of the TN FERTINNOWA, a series of Facebook posts were created to be released during the next five months after the end of the project. This allowed them to further disseminate TN results and resulted in an increase in followers of the webpage. As a consequence, this may also increase the number of views per video [29]. However, the use of social media is time-consuming, and TN partners are not using them enough, slowing down the development of the digital community and the diffusion and promotion of the videos [28]. It should be noticed that the use and application of social media may strongly depend on other factors as well, such as broadband connectivity, digital skills, and the social and cultural background of $\mathrm{TN}$ partners that varies from region to region within Europe. Thus, there is a need for a more in-depth study of the use of social media as an efficient C\&D tool for farmers, foresters, and advisors.

TNs should have regular (e.g., daily) activity (posts, shares, likes) in order to reach at least 500 followers and create a good engagement rate [30]. This allows them to engage followers through social networks and creates benefits for project communication and dissemination. More activities and posts through a social network may result in more followers, and consequently, in more views and impact. TN partners and other actors should be made aware of the importance of having a well-defined strategy of spreading videos to their networks and their peers. A possible reason why videos have a low number of views may be that they were not accurately disseminated. However, Alföldi et al. [26] stated that, "depending on the topic, the quality of the videos produced, and the existing networks, it can take months, if not years, for a new YouTube channel to be accepted by the audience".

Nevertheless, even though the average number of views of the analyzed videos is relatively low, there are a few outliers with more than 10,000 views. These are showcase videos. The reasons for the popularity of these few videos may be attributed to promotional events in which TNs took part or accurate dissemination through the use of other channels such as TN newsletters and social media.

\subsection{Content Type}

The content type may be one of the reasons for the disparity in the number of views between videos. Figure 3 shows a boxplot of the number of views in relation to the content type. Presentations and videos with question and answer sessions generally have fewer views than the other content type categories. This is confirmed by Kendall's rank correlation coefficient between the videos' number of views and whether or not it is a presentation, which is approximately $-0.43(z=-11.786)$, suggesting a moderate correlation. A similar relationship can be found with the average percentage viewed for the subset of TNs, as the correlation coefficient is approximately -0.26 . Similar observations were found when inspecting the content type of each individual TN's videos. 


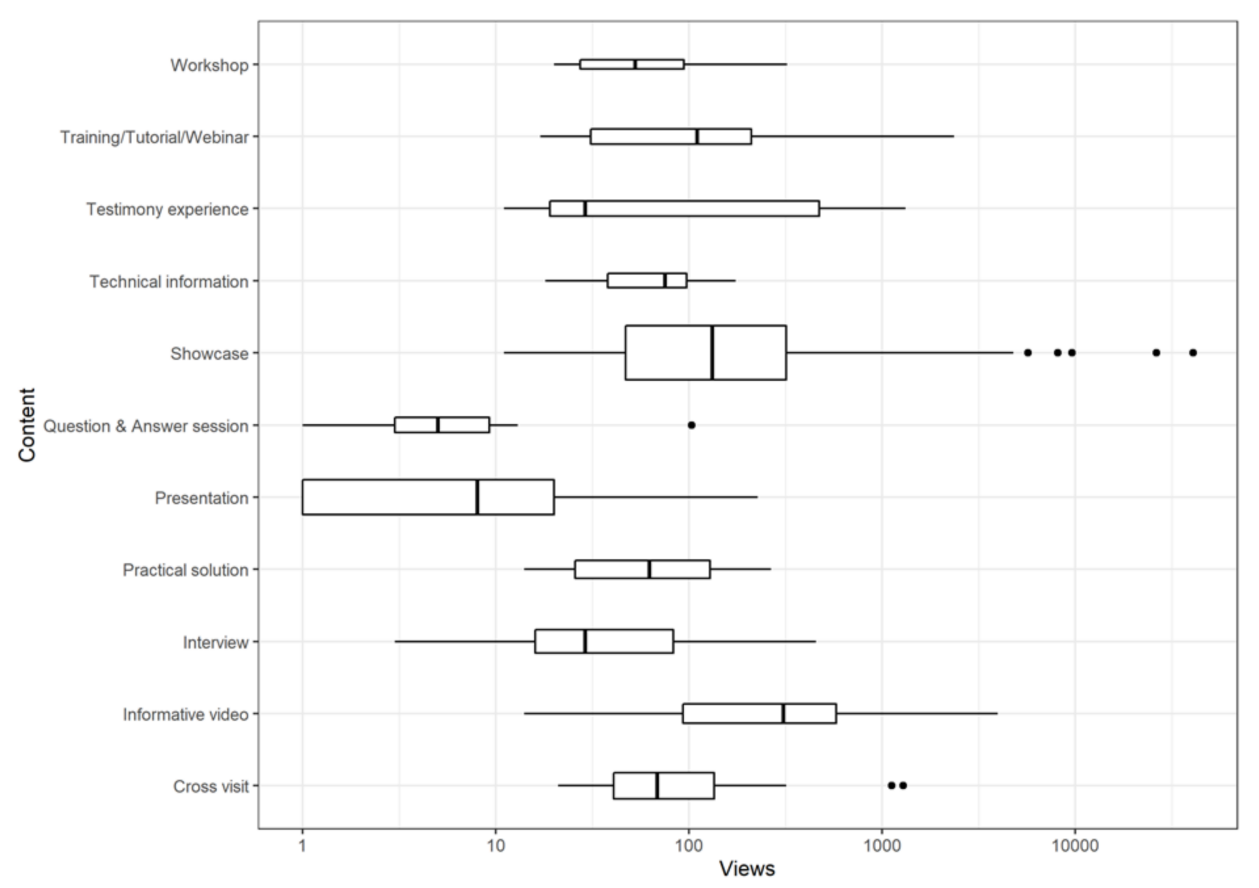

Figure 3. Number of views in relation to the content type.

Showcases are the most common content type $(42 \%)$, with a median number of views of 132, although some showcases videos that have more than 10,000 views. As outputs of their work, TNs also produced videos related to other activities such as crossexchange visits (10\% of all videos) and interviews with coordinators/farmers/advisors ( $8 \%$ of all videos).

The fact that showcases represent the most-used typology of videos is in line with the purpose of a TN. That is, the purpose is mainly collecting and translating existing knowledge and transforming it into ready-to-use solutions for practitioners. Creating videos to demonstrate the range of available information in a specific farming and food sector may also encourage students to consider a future in the sector [31]. This aspect is particularly important in TNs, as the majority of them struggle to engage students and vocational schools [15,32].

A study from Van Mele [33] and Van Campenhout, Spielman, and Lecoutere [23] proved that peer-to-peer communication on practical field solutions could lead to a faster result uptake among farmers that are keener to apply new technologies or field techniques. This was also confirmed from the outcomes of the interviews performed in the EURAKNOS project. Here, $94 \%$ of the TNs coordinators and C\&D experts believe that a video should contain practical content, such as a field demonstration. However, $68 \%$ believe it is also important to have scientific content. Related to the format, $65 \%$ would prefer an interview recording included in the video [28]. However, there are many videos with a low number of views in all content type categories. This indicates that simply producing these videos is not sufficient. They need to be spread more to increase the chance of being used and applied in the field. Since the most-watched videos are showcases, and their importance and efficiency are well documented in the literature [34], TNs should put more effort into producing this type of video. For example, they can invest more budget to create a high-quality final product. Combined with an efficient C\&D strategy and farmers' / foresters' involvement throughout the project, this may significantly improve the uptake of the message they want to convey.

\subsection{Speaker}

Ensuring farmers' attention and willingness to use TN results may be enhanced by the presenter or the actors that are involved in the video. The benefit of having farmers involved 
as a speaker is widely reported in the literature [35]. Based on a study by Harwin and Gandhi [14], "farmers will pay close attention to the presenter of a video, even down to the outfit worn to determine whether they trust the video". The box plots in Figure 4 show that the information regarding the role of speakers in many $\mathrm{TN}$ videos was not available (44\%). When they were available, farmers and researchers were the most common categories $(25 \%$ and $17 \%$, respectively). The other categories are so uncommon (less than $3 \%$ per category) that drawing conclusions from them is not possible. Students were a non-represented category. Once again, this confirms the lack of involvement of student and vocational schools in TN activities.

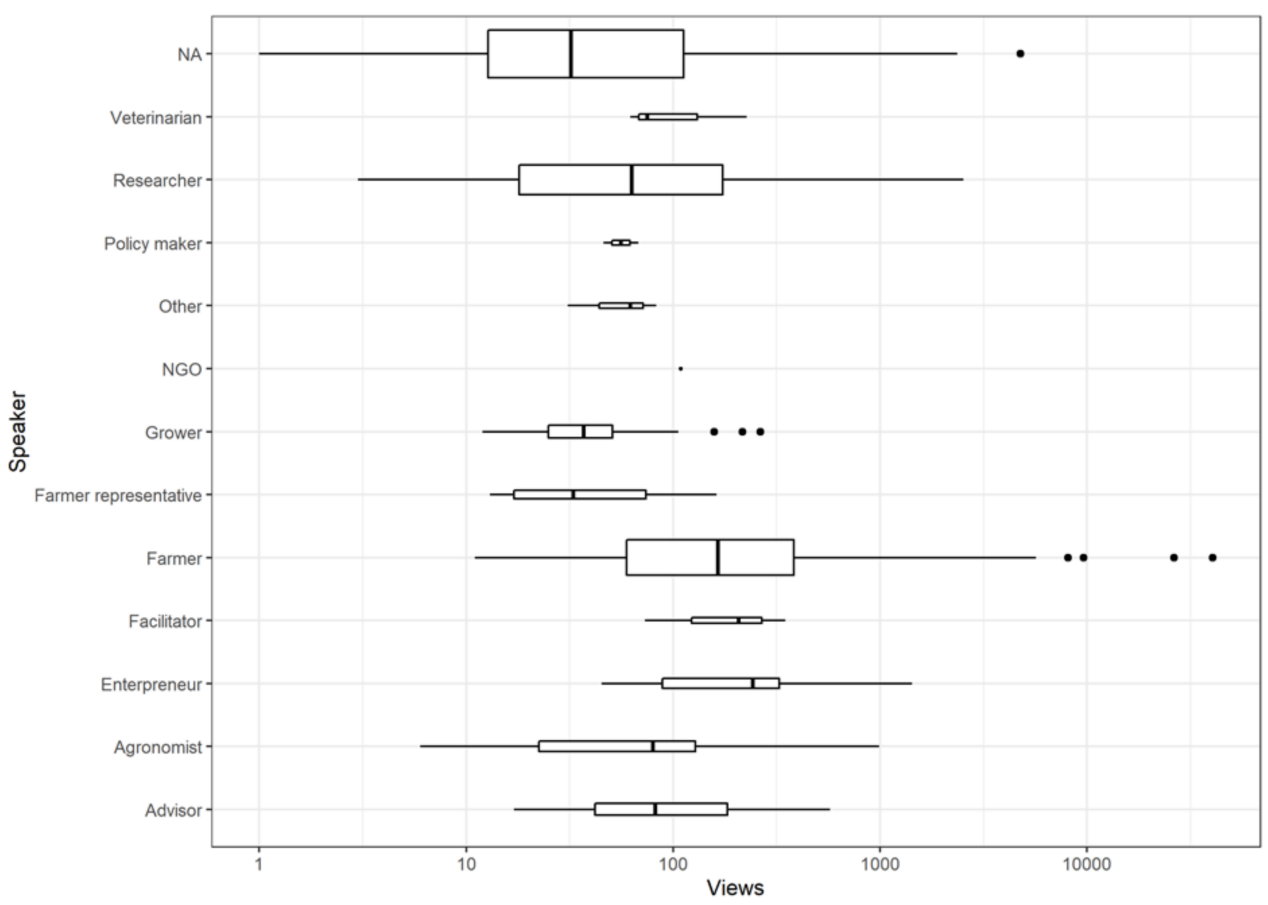

Figure 4. Number of views in relation to who is speaking in the video.

Results indicate that farmers generate more views than researchers (median of 165 views compared to 63 views). This is confirmed by Kendall's rank correlation coefficient between the videos' number of views and whether or not the speaker is a farmer (calculated over only videos with farmers or researchers as speakers). The correlation is approximately $0.24(z=4.2647)$, which suggests a moderate correlation. A similar relationship can be found with the average percentage viewed for the subset of TNs, as the correlation coefficient is approximately 0.27 . We found a similar relationship when investigating each TN individually. Note that this relationship is influenced because researchers are often the main speaker in (long) presentation videos, which are viewed much less than other content types (as discussed in Sections 3.5 and 3.8).

Not only is the person speaking in the videos relevant, but so are the TN partners that were in charge of making (and disseminating) the video. For example, TNs should implement the involvement of advisors in the video-making process [13]. In fact, by closely working with farmers, advisors can easily promote $\mathrm{TN}$ videos and knowledge, enhancing the use of the information that TN outputs want to convey. Unfortunately, the information about which TN partner was in charge of making the video was mostly not available. Future TNs should include more metadata, such as the creator of the video, date of creation, and a short content description with keywords. In this way, videos can be more easily found and consulted, and the knowledge can be applied even after the end of TN projects.

The selection of the speaker in the videos may also need to be adapted to the audience that these videos aim to reach. This is usually defined properly in the C\&D plans at the beginning of the project. Project partners in charge of the video production should take the 
behavior and preferences of the different target audiences into account. For example, how they obtain knowledge and which is the best way to catch their attention. For example, if the video is a recorded presentation or webinar, it has been proposed to have farmers in the speaker panel in order to gain the participants' trust [34]. This strategy can differ according to the agricultural sector, gender, age, culture, country, or political framework. In this way, they can make a tailor-made and effective video.

In conclusion, videos that have farmers as speakers are the ones that generally generated the largest number of views. This aspect should be taken into account in future TNs, since farmers tend to trust information acquired in videos where other peers or people with similar skills are presenting [23]. Furthermore, engaging farmers/foresters in videos is a way of keeping their involvement and stimulating their interest in the work of a TN. This will also show that they can concretely benefit from the results and best practices that the $\mathrm{TN}$ is producing.

\subsection{Language: Number of Subtitles}

The language that is used represents an important factor that TNs need to take into account. What emerged in the analysis, which was made in the EURAKNOS project, is that TNs tried to be as accurate as possible in adopting a clear language for users in their outputs. However, it remains an important barrier to properly reach, communicate with, and disseminate the expected targets. Once the audience, message, and the right strategy of engagement is identified, videos should be translated in the language of actors that were, directly and indirectly, involved in the TN's activities. This enables cross-cultural communication in different countries [36,37].

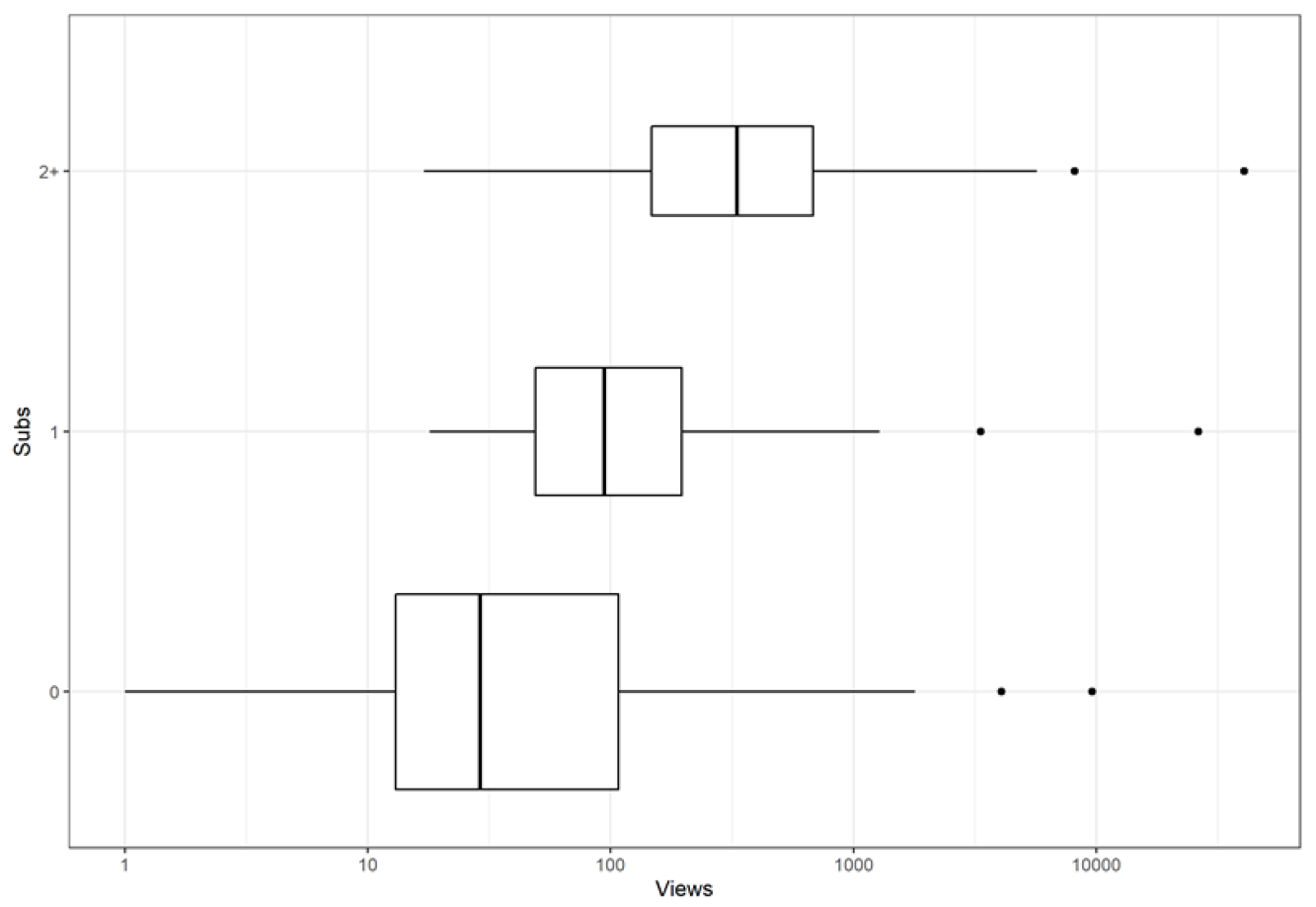

Figure 5. Number of views in relation to the number of subtitles.

The language aspect was investigated in Figure 5, which shows a boxplot of the number of views in relation to the number of subtitles. The figure shows that a larger number of subtitles (i.e., more than two different languages) results in a higher number of views (i.e., a median number of views of 331 for 2+ subtitles, compared to 94 and 29 for 1 and 0 subtitles, respectively). This is confirmed by Kendall's rank correlation coefficient between the video's number of views and the number of subtitles, which is approximately $0.39(z=11.397)$, suggesting a moderate correlation. No correlation is found with the average percentage viewed for the subset of TNs, as the correlation coefficient 
is approximately -0.11 , and the $p$-value is 0.17 . However, the majority of the videos are without any subtitles or only have one language for subtitles.

We performed this analysis for each TN individually as well and found the same relation between the number of subtitles and views. Some exceptions to this are some testimony experience videos of the TN CERERE. These videos have a single subtitle but were watched less than the videos of that TN with 0 subtitles, which could, for example, be due to them being promoted less.

The lack of subtitles represents a limitation for efficient uptake and spread of the results. By adding subtitles to videos, a TN can enhance the creation of a learning environment. If the translation of the content of the video is missing, only actors that speak that specific language can benefit from the knowledge available in a video. This limits the diffusion of innovation and increases the knowledge gap at the European level. Considering this barrier, TN's C\&D plans must be foreseen to directly reach the target with the project outputs.

In general, subtitles have several advantages. For instance, they are a helpful tool for translating the video in many languages, and they can help the person who is watching to keep their attention on the message. Additionally, subtitles make a video accessible for those who do not hear or have a low hearing. If users find that a video is easier to understand due to the available subtitles, they are more likely to share and use the video, which expands the audience [12]. Cuendet et al. [38] found that, in countries with low-literacy level farmers, tools providing speech-based interfaces to present videos in local languages failed to become fully inclusive. However, since the audience of TNs is mostly at the European level, the level of literacy of farmers is sufficiently high to read subtitles, even in remote rural areas [34]. Another aspect related to reaching remote rural areas may be poor digital connectivity. As Lyon et al. [39] pointed out, digital divides persist in some rural communities in Europe (e.g., in England) due to a lack of internet connection or slow broadband. It is therefore crucial that the language used in videos remains simple and easily understandable. Based on the work by Rose [34], an efficient video is not overloaded with technical jargon, and the length should be long enough to convey useful information. Although one should be careful with longer videos, as this may lead to audience disengagement. This aspect is investigated in Section 3.8.

\subsection{Video Length}

The boxplots in Figure 6 show the result of the analysis of the length of TNs videos. The majority of the videos (56\%) fall in the category $1-5 \mathrm{~min}$. Overall, $10 \%$ fall in the category $0-1 \mathrm{~min}, 15 \%$ in the category $5-10 \mathrm{~min}$, and $19 \%$ are longer than $10 \mathrm{~min}$.

We can observe that many videos that are longer than 10 min have few views. This is confirmed by Kendall's rank correlation coefficient between the video's number of views and whether or not the video is longer than $10 \mathrm{~min}$, which is approximately -0.32 $(z=-8.9123)$, suggesting a moderate negative correlation. A similar relationship can be found with the average percentage viewed for the subset of TNs, as the correlation coefficient is approximately -0.22 .

We found similar observations when investigating each TN individually. It should be noted, though, that most videos in the category of videos longer than $10 \mathrm{~min}$ are presentations. As discussed in Section 3.5, these are generally less watched. Therefore, when TNs produce videos on conferences, presentations or question and answer sessions, it is advised to make concise videos that only capture the most relevant moments [34]. In this way, the target audiences that were not able to attend the event can remain engaged more efficiently. 


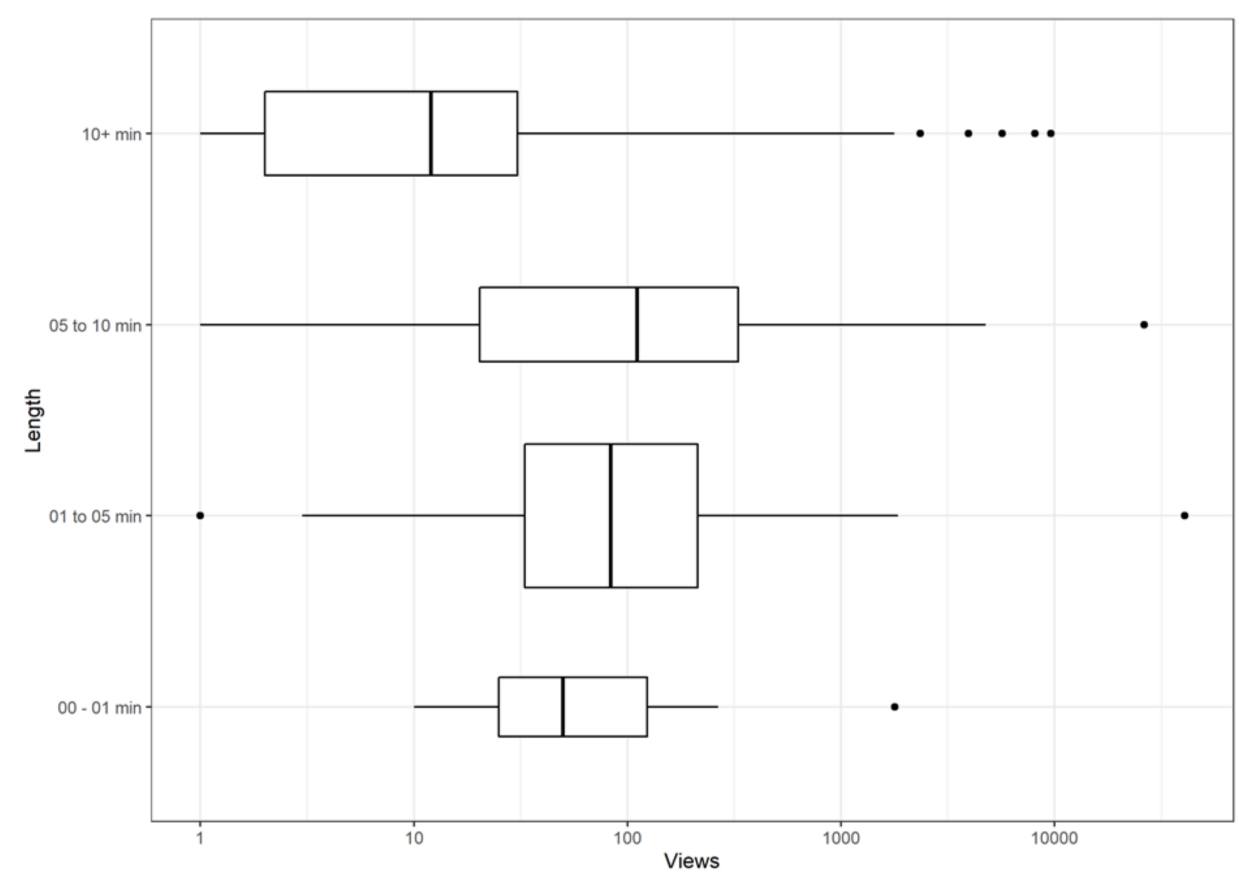

Figure 6. Number of views in relation to the video length.

On the one hand, having concise videos of less than 10 min allows having a high engagement rate. On the other hand, short videos contain less information. The study of Bliss et al. [22] highlighted that short videos may fail to provide enough detail to encourage practical application. Furthermore, due to their relatively fast-paced nature, the details may not be grasped [40]. However, TNs should use short videos to trigger the curiosity of the audience, which may be stimulated to dig deeper into the TN results. Long videos may have the same low engagement effect, especially if it is a recording of a conference or presentation. Moreover, it is well known that farmers have a limited amount of time to spend in front of a computer; hence, a short video that tackles the most relevant aspect may increase its efficiency. When it comes to TNs, based on the results in Figure 6, videos should be kept short (no longer than $10 \mathrm{~min}$ ), and the key message should be conveyed at the beginning of the video. This is supported by Wright et al. [41], who suggest that, "if greater detail is required, the video could link to further resources (including additional videos) for viewers who wish to engage further". TNs should then focus more on producing a teaser video (to communicate about new project outcomes) that contains a link to other longer videos where they disclose the results.

\section{General Discussion}

This section discusses how to increase the uptake of $\mathrm{TN}$ videos results through the use of a centralized knowledge platform.

\subsection{Communication and Dissemination Channel through Knowledge Platform}

It is also important to note that the information reported in $\mathrm{TN}$ videos may be misinterpreted [42]. To face this, TNs should present those videos in events where experts are present (e.g., workshops) and foresee time to discuss the content (as should also be done during online meetings). More generally, even though videos are successful tools to exchange knowledge, it remains fundamental to combine them with more "traditional" ways of knowledge exchange (e.g., physical meetings). Videos should never be used as an exclusive tool, and the combination of both ways of exchanging knowledge is the most likely way to encourage farmers to translate knowledge into action [22]. TNs are already successfully promoting face-to-face exchange moments. 
What may enhance the number of views of videos, and hence the uptake of the results, is the creation of a knowledge platform where all videos and other relevant outcomes produced by TNs are stored. In fact, as reported by Chourt and Pascal [43], platform-related technologies can play a catalytic role in the dissemination of knowledge. However, despite a large number of existing agriculture-related platforms, the content provided by most of them does not meet the needs of all the user types [12]. The EURAKNOS project studied the feasibility of creating such a user's platform where the knowledge that TNs collected is stored [12]. In line with AKIS strategies and the CAP, the creation of an EU-wide knowledge platform that collects all-ready-for-practice materials from all TNs will help to create an EU agriculture and forestry community, strengthening the MAA and AKIS. Additionally, links between research, practice, education, and advisors may be easily made and knowledge is potentially more efficiently exchanged outside the national borders. Since 2014, the EU has invested in a large number of agriculture-based support and innovation actions to improve knowledge flows between projects and actors. Through the EURAKNOS and the follow-up project EUREKA [44], the EU is investing more than 4 million euros to make this open-source knowledge platform (the FarmBook) a concrete tool.

A knowledge database can allow users to have access to relevant networks of actors. Furthermore, it can allow having direct interaction with farmers, so that they can communicate their needs. Older farmers may need an intermediary, such as an advisor, or someone younger in the family (e.g., a son or nephew) to help them decide which TNs knowledge is relevant and how they can implement it. Hence, it is important to have digitally trained advisors and other key intermediary actors. However, to be successful and more impactful, the content needs to be well-structured, and the interface of such a digital platform needs to be strongly user-oriented.

Even though the knowledge database proposed in the EURAKNOS project is structured to serve TN users' needs, it can also have an impact at the political level. Policymakers can close the gap between policy at the regulatory level and practice, enhancing the effective use of TNs outputs. However, adequate financial resources should be foreseen to maintain the platform over time to guarantee its dynamicity [45].

\subsection{Quality Control and Impact}

The adoption and use of a knowledge platform are conditioned by the quality and relevance of stored data. Hence, validation of content and quality control need to be ensured. Regarding this last aspect, based on the performed analysis in this paper, not all videos produced by TNs are interesting in the way they are presented. For example, conference recordings could be edited in a way to capture the most relevant part and not simply show the recording of the whole event. Guidelines on how to make impactful videos without expensive equipment are made available by several multi-actor H2020 projects (e.g., the PLAID and NEFERTITI projects). Given the fact that future TNs will foresee the realization of videos, the guidelines provided from those projects should be followed and applied.

Another aspect that future TNs should take into account is to monitor the real impact of their videos. For example, by checking whether the results that videos convey are concretely applied by farmers or foresters. This can be done in case a follow-up project is granted. In other words, has the solution provided in those videos been concretely used? The answer is not straightforward. In this context, YouTube analytics can offer a point of reflection. By knowing the "average percentage viewed", the owner of the YouTube channel (in this specific case the TN coordinator or the partner in charge of the C\&D) has access to the average time that viewers watched each video. This means that the video's ability to hold viewers' attention for its entirety is measured. Furthermore, when YouTube realizes that a certain video can hold people's attention, it is rewarded with higher search and recommendation rankings [46]. Unfortunately, those analytics were not taken into account in the final reports of the 16 analyzed TNs. This study analyzed the average percentage viewed of some TNs but failed to receive these private analytics of all TNs. 
In conclusion, measuring the impact represents a difficult aspect in TNs. Follow-up projects may investigate whether the results presented in videos were applied in practice. Moreover, it should be validated if they brought an improvement in the activity of actors that used these results. This can be investigated with surveys, meetings, workshops, and the use of analytics tools.

\section{Conclusions}

The outputs of TNs have a high potential impact to farmers/foresters and other actors. That is, they stimulate the practical implementation of sustainable and innovative solutions in agriculture and forestry. Videos are an important tool that TNs use to disseminate and communicate about the projects and their outcomes. Even though TNs create a detailed C\&D plan to reach a wide public, our study highlights that the majority of produced videos have a low number of views. In other words, the high potential practical impact is not always reached. Thus, TNs should spend more attention in their C\&D plan on how to create high-quality videos in order to increase their impact.

In order to suggest best practices for videos of future TNs, we investigated the characteristics of 517 videos produced by 16 TNs. More importantly, we analyzed the impact of these characteristics on the number of views and average percentage viewed.

A first interesting observation that was made is that quantity does not equal quality. That is, producing more videos does not necessarily mean that they will be watched more. In fact, we even observed a slight negative correlation between the number of videos made by a TN and their number of views. Additionally, we noticed that videos produced at the end of a project's lifespan are often less viewed than those made at the beginning. This could be due to them having less time to watched, but also because they might be promoted less. Related to this, we noticed that videos produced for communication process were scarce, yet generally watched more than those made for dissemination purposes. As such, TNs should consider producing more videos that communicate about their project, as this may stimulate the audience's curiosity to further investigate TN results.

Another interesting observation is that (long) presentations are generally watched less than other content types. As such, we suggest that authors focus more on showing practical content (such as showcases and training/tutorial videos). In general, future TNs should edit recordings of events. TNs should create a short key message to trigger the attention of the audience. As a result, the audience will be more stimulated to watch these short videos, and the key message will be more easily conveyed. Again, these short videos may be a gateway to longer videos, where TNs disclose their results in more depth.

Not only are the content type and length of TN videos important, but so is the speaker. We found that videos featuring farmers as speakers are often viewed more than videos with other speakers. We suggest that TNs take this into account, although the speaker may also need to be adapted to the audience that these videos aim to reach.

The language remains a barrier as well, as the majority of the videos are not translated or subtitled. However, we found that videos with more subtitles are generally watched more. Thus, future TNs should make an effort to translate their videos in order to reach a wider audience.

The best practices proposed in this study are particularly interesting for the common EU-wide agricultural knowledge platform that the EU Horizon2020 EURAKNOS and EUREKA projects are building. That is, having well-edited videos on the platform may improve the impact of TNs results. Such videos have the ability to disclose important knowledge and good practices that will help to enhance agricultural and forestry sustainability. It would also mean that target users can more easily find information and provide feedback in one place, resulting in higher web traffic compared to individual project websites. It is, however, essential that videos are well described by metadata. The community of actors that the common knowledge platform will create suits the AKIS strategies for improving information flows within the system. It is essential for generating innovation, as well as building and sharing knowledge openly. Additionally, it creates a space for actors to 
meet and develop ideas together, addressing common problems. Without substituting full face-to-face activities, a well-maintained platform adapts to user needs and expectations.

During this study, we noticed that measuring the effective uptake of video results from farmers and foresters is not a straightforward process. The number of views of the videos is publicly available for all TNs, but more-detailed statistics such as the average percentage watched are only available to the YouTube channel owner. Although we were able to analyze this private statistic for few TNs, future TNs should use YouTube Analytics as a tool to better measure the level of involvement of the watcher. This may allow them to improve their dissemination and, in cases of follow-up projects, to better monitor whether practical solutions have been applied in the field.

The use of new technologies and new digitized ways of interactions among actors will need to be investigated in future research. Possible sets of future research questions include how the use of digital tools can affect farming activities, as well as their influence on farmers' learning, experiences, and skills.

In conclusion, videos made by TNs aim to strengthen the links between research, practice, education, and farm advisors. This study aids the creation of high-quality videos in order to stimulate interactive and sustainable innovation in practice. Additionally, it supports the digital transition in agriculture and forestry.

Author Contributions: Conceptualization, S.B., P.S. and E.F.; methodology, S.B., P.S. and E.F.; software, H.M. and E.F.; validation, H.M. and E.F.; formal analysis, H.M. and E.F.; investigation, E.F.; resources, E.F.; data curation, H.M. and E.F.; writing-original draft preparation, E.F.; writingreview and editing, H.M., S.B. and P.S.; visualization, H.M. and E.F.; supervision, S.B. and P.S.; project administration, S.B. and P.S.; funding acquisition, S.B. and P.S. All authors have read and agreed to the published version of the manuscript.

Funding: The paper is funded under the EURAKNOS project. The EURAKNOS project has received funding from the European Union's Horizon 2020 research and innovation program under grant agreement No: 817863 . Additionally, this work was partly funded by the Research FoundationFlanders (FWO) under Grant 1S55218N.

Institutional Review Board Statement: Not applicable.

Informed Consent Statement: Not applicable.

Data Availability Statement: The data used in this study is available on https://cloud.ilabt.imec. be/index.php/s/giLggrmjpHmbRQy (accessed on 10 October 2021).

Conflicts of Interest: The authors declare no conflict of interest.

\section{Appendix A}

Table A1. Video categorization based on their characteristics.

\begin{tabular}{|c|c|}
\hline Characteristics of the Videos & Description \\
\hline Number of views & How many times the video has been watched \\
\hline Average percentage viewed & How long the viewers watched the video, on average \\
\hline Topic (theme of the TN) & Livestock, forestry, and crop farming \\
\hline Release date & $\begin{array}{l}\text { When the video was uploaded, relative to the project time span } \\
\text { (beginning/middle/end of the project) }\end{array}$ \\
\hline Dissemination or Communication & $\begin{array}{l}\text { The video was made to either communicate about the project } \\
\text { or to disseminate the results/best practices }\end{array}$ \\
\hline Content-type & $\begin{array}{l}\text { Workshop, training/tutorial/webinar, testimony experience, technical information, } \\
\text { showcase, question and answer session, presentation, interview, informative video, } \\
\text { cross-exchange visit }\end{array}$ \\
\hline Speaker & Which main actor (e.g., farmer, advisor, researcher) was speaking \\
\hline Partner Role & TN's partner in charge of the video making \\
\hline Language & Which language is available in the video \\
\hline Number of subtitles & How many subtitles are available in the video \\
\hline Video duration & How long is the video duration \\
\hline
\end{tabular}


Table A2. Video subdivision and relative description.

Type of Video

Workshop

Training/tutorial/webinar

Testimony experience Technical information

Showcase

Question and answer session

Presentation

Practical solution

Interview

Informative video

Cross-exchange visit

\section{Description}

Moments and highlights of workshops organized by TNs with farmers/foresters and other actors

Videos that are shot on site that describe how

to conduct a specific activity or use a specific technique/machine/tool; the explanation is with technical details

Actor's statement regarding the use of a specific technique/tool/methodology A technical video presents the salient aspects of a project in an engaging way, or a piece of machinery, without going too much into any details covered by industrial secrecy or patents

Videos that aim to show a specific activity held in a farm/field, without providing technical details

A period of time or an occasion when TN coordinator or other actors involved in TN activities answers questions that are asked by a reporter

Recordings of (online) presentations

Example of solutions to a problem/difficult situation, and the way of dealing with it

Formal consultation with farmers/foresters, advisors, researchers, project partners and coordinators on a given TN theme

Teaser videos are used to promote the project or specific TN outcomes

Moments and highlights of cross-exchange visits organized by TNs with farmers/foresters and other actors from the different EU States

\section{References}

1. Coffey International. Evaluation Study of the Implementation of the European Innovation Partnership for Agricultural Productivity and Sustainability; Coffey International: Berkshire, UK, 2016.

2. Ruoslahti, H. Complexity in project co-creation of knowledge for innovation. J. Innov. Knowl. 2020, 5, 228-235. [CrossRef]

3. Faure, G.; Knierim, A.; Koutsouris, A.; Ndah, H.T.; Audouin, S.; Zarokosta, E.; Wielinga, E.; Triomphe, B.; Mathe, S.; Temple, L.; et al. How to Strengthen Innovation Support Services in Agriculture with Regard to Multi-Stakeholder Approaches. J. Innov. Econ. 2019, 28, 145-169. [CrossRef]

4. Fieldsend, A.F.; Cronin, E.; Varga, E.; Biró, S.; Rogge, E. Organisational Innovation Systems for multi-actor co-innovation in European agriculture, forestry and related sectors: Diversity and common attributes. NJAS Wagening. J. Life Sci. 2020, 92, 1-11. [CrossRef]

5. Feo, E.; Burssens, S.; Berckmoes, E.; Pascal, E.; Mosquera-losada, R. The Multi-Actor Approach in Thematic Networks for Agriculture and Forestry Innovation. 2021, 1-36, in press.

6. EURAKNOS. Deliverable D3.3-WP3. HIKR Communication, Dissemination E Exploitation Strategies; EURAKNOS: Gent, Belgium, 2020.

7. Marín-González, E.; Malmusi, D.; Camprubí, L.; Borrell, C. The Role of Dissemination as a Fundamental Part of a Research Project: Lessons Learned from SOPHIE. Int. J. Health Serv. 2017, 47, 258-276. [CrossRef]

8. Scherer, J.; Weber, S.; Azofra, M.; Ruete, A.; Sweeney, E.; Weiler, N.; Sagias, I.; Haardt, J.; Cravetto, R.; Spichtinger, D.; et al. Making the Most of Your H2020 Porject. Boosting the Impact of Your Project through Effective Communication, Dissemination and Exploitation; European IPR Helpdesk: Luxemburg, 2018; 36p.

9. Lee, S.Y.; Díaz-Puente, J.M.; Vidueira, P. Enhancing rural innovation and sustainability through impact assessment: A review of methods and tools. Sustainability 2020, 12, 6559. [CrossRef]

10. European Commission. Annotated Model Grant Agreement; European Commission: Luxembourg, 2015.

11. European Commission. Horizon Europe Programme Guide-EU Grants: HE Programme Guide: V1.1; European Commission: Luxembourg, 2021.

12. EURAKNOS. Deliverable 4.1-WP4. Report on the Added Value and Feasibility of Developing a New Standardised e-KRP Based on End-User Needs; EURAKNOS: Gent, Belgium, 2019.

13. Bentley, J.W.; van Mele, P.; Barres, N.F.; Okry, F.; Wanvoeke, J. Smallholders download and share videos from the Internet to learn about sustainable agriculture. Int. J. Agric. Sustain. 2019, 17, 92-107. [CrossRef]

14. Harwin, K.; Gandhi, R. Digital Green: A Rural Video-Based Social Network for Farmer Training (Innovations Case Narrative: Digital Green). Innov. Technol. Gov. Glob. 2014, 9, 53-61. [CrossRef]

15. Feo, E.; Bodin, P.; Burssens, S.; Mosquera-Losada, R.; Tourner, L.; Guichaoua, A.; Duschesne, R.; Spanoghe, P. Communication, Dissemination, and the use of social media in H2020 Thematic Networks. In International Agriculture Symposium; University of East Sarajevo: Republika Srpska, Bosnia and Herzegovina, 2020; pp. 1-6. 
16. van Mele, P.; Okry, F.; Wanvoeke, J.; Barres, N.F.; Malone, P.; Rodgers, J.; Rahman, E.; Salahuddin, A. Quality farmer training videos to support South-South learning. CSI Trans. ICT 2018, 6, 245-255. [CrossRef]

17. SCAR AKIS. Preparing for Future Akis in Europe-4th Report of the Strategic Working Group on Agricultural Knowledge and Innovation Systems. 2019. Available online: https://scar-europe.org/index.php/news-display/173-4-report-akis (accessed on 10 October 2021).

18. Schut, M.; Klerkx, L.; Kamanda, J.; Sartas, M.; Leeuwis, C. Innovation Platforms: Synopsis of Innovation Platforms in Agricultural Research and Development. In Reference Module in Food Science; Elsevier: Amsterdam, The Netherlands, 2018 ; pp. 510-515.

19. EIP-AGRI. EIP-AGRI Seminar CAP Strategic Plans: The Key Role of AKIS in Member States. 2020. Available online: https: / / ec.europa.eu/eip/agriculture/en/event/eip-agri-seminar-cap-strategic-plans-key-role-akis (accessed on 10 October 2021).

20. European Commission. Proposal for a regulation of the European Parliament and of the Council establishing rules on support for strategic plans to be drawn up by Member States under the Common agricultural policy (CAP Strategic Plans) and financed by the European Agricultural. Off. J. Eur. Union 2018, 0216, 143.

21. Sousa, F.; Nicolay, G.; Home, R. Video on Mobile Phones as an Effective Way to Promote Sustainable Practices by Facilitating Innovation Uptake in Mali. Int. J. Sustain. Dev. Res. 2019, 5, 1-8. [CrossRef]

22. Bliss, K.; Padel, S.; Cullen, B.; Ducottet, C.; Mullender, S.; Rasmussen, I.A.; Moeskops, B. Exchanging knowledge to improve organic arable farming: An evaluation of knowledge exchange tools with farmer groups across Europe. Org. Agric. 2019, 9, 383-398. [CrossRef]

23. van Campenhout, B.; Spielman, D.J.; Lecoutere, E. Information and Communication Technologies to Provide Agricultural Advice to Smallholder Farmers: Experimental Evidence from Uganda. Am. J. Agric. Econ. 2021, 103, 317-337. [CrossRef]

24. Sodagar, H.R.; Es'hagh-Pour Rezaee, M.; Rezaie, H. Forester Navigation Application (FNA): Mobile Training in Environmental Organizations. Interdiscip. J. Virtual Learn Med. Sci. 2021, 12, 73-75.

25. Karubanga, G.; Agea, J.G.; Okry, F.; Kiwewesi, S.; Mugerwa, J.L.K. Factors effecting change in rice production practices and technologies among smallholder farmers in kamwenge district, Uganda. Indian J. Ecol. 2019, 46, 316-324.

26. Alföldi, T.; Tippin, L.; Midmer, A.; Hardy, C.; Vanev, D. Video Production for Agriculture A Guide for Farmers, Advisors and Researchers. 2019. Available online: https://orgprints.org/id/eprint/37544/1/alfoeldi-etal-2019-Videoguide_EN_25June201 9.pdf (accessed on 10 October 2021).

27. EIP-AGRI. Available online: https:/ / ec.europa.eu/eip/agriculture/en/find-connect/projects (accessed on 10 October 2021).

28. EURAKNOS. Deliverable 2.3-WP2. Report on Used Communication and Dissemination Tools, and Information Channels of TNs; EURAKNOS: Gent, Belgium, 2020.

29. Barau, A.A.; Afrad, M.S.I. An overview of social media use in agricultural extension service delivery. J. Agric. Inform. 2017, 8, 50-61. [CrossRef]

30. Jaakonmäki, R.; Müller, O.; vom Brocke, J. The Impact of Content, Context, and Creator on User Engagement in Social Media Marketing. In Proceedings of the 50th Hawaii International Conference on System Sciences, Waikoloa, HI, USA, 4-7 January 2017.

31. Dodson, E.M.; Blinn, C.R. Forest operations instructor and student perspectives on rapid transition from face-to-face to online learning in the US. Int. J. For. Eng. 2021, 1-11. [CrossRef]

32. Cooreman, H.; Debruyne, L.; Vandenabeele, J.; Marchand, F. Power to the facilitated agricultural dialogue: An analysis of on-farm demonstrations as transformative learning spaces. J. Agric. Educ. Ext. 2021, 27, 699-719. [CrossRef]

33. van Mele, P. Video-Mediated Farmer-to-Farmer Learning for Sustainable Agriculture A Scoping Study for SDC, SAI Platform and GFRAS. 2011. Available online: https:/ / agroinsight.com/downloads/articles-divers/Farmer-to-farmer-video-FINALREPORTVan-Mele-2011.pdf (accessed on 10 October 2021).

34. Rose, D.C.; de Boon, A.; Schillings, J. Videos and Podcasts as Potential Approaches for Knowledge Exchange with Farmers: Testing Their Potential Role in ELM; Results from a Literature Review and an Empirical Study in England; University of Reading: Reading, UK, $2021 ; 57 p$.

35. Bentley, J.; Chowdhury, A.; David, S. Videos for Agricultural Extension. GFRAS Platform. 2015. Available online: https: / / www.fao.org/3/i5020e/i5020e.pdf (accessed on 10 October 2021).

36. Bentley, J.; van Mele, P. Sharing ideas between cultures with videos. Int. J. Agric. Sustain. 2011, 9, 258-263. [CrossRef]

37. Fry, P.; Thieme, S. A social learning video method: Identifying and sharing successful transformation knowledge for sustainable soil management in Switzerland. Soil Use Manag. 2019, 35, 185-194. [CrossRef]

38. Cuendet, S.; Medhi, I.; Bali, K.; Cutrell, E. VideoKheti: Making video content accessible to low-literate and novice users. In Proceedings of the Conference on Human Factors in Computing Systems, Paris, France, 27 April 2013; 2013; pp. $2833-2842$.

39. Lyon, J.; Hurley, P.; Hall, J.; Tsouvalis, J.; Rose, D.C.; Little, R. Inclusive Design of Post-Brexit Agri-Environmental Policy: Identifying and Engaging the 'Harder to Reach' Stakeholders A Quick Scoping Review; The University of Sheffield: Sheffield, UK, 2020.

40. Burbi, K.H.; Rose, S. The role of internet and social media in the diffusion of knowledge and innovation among farmers. In Proceedings of the 12th European IFSA Symposium, Newport, UK, 12-15 July 2016.

41. Wright, D.; Hammond, N.; Thomas, G.; MacLeod, B.; Abbott, L.K. The provision of pest and disease information using Information Communication Tools (ICT) an Australian example. Crop Prot. 2018, 103, 20-29. [CrossRef]

42. Vasilaky, K.; Toyama, K.; Baul, T.; Mangal, M.; Bhattacharya, U. Learning Digitally: Evaluating the Impact of Farmer Training via Mediated Videos. Inf. Technol. Int. Dev. 2015, 5, 1-27. 
43. Chourt, J.-M.; Pascal, E. CASA-Deliverable 4.2: Report of Best Practices from Swg Scar Akis Members. 2018. Available online: https:/ / scar-europe.org/images / AKIS/Report_Meetings/SCAR-SWG-AKIS4_Report-10-Brussels.pdf (accessed on 10 October 2021).

44. EUREKA. Available online: https:/ / cordis.europa.eu/project/id/862790 (accessed on 10 October 2021).

45. Burssens, S.; Palczynski, L.; Rasmussen, I.; Stock, A. Sustainability of Thematic Networks for Agricultural and Forestry Innovation: Recommendations from the EURAKNOS Project. 2020. Available online: https://euraknos.fra1.digitaloceanspaces.com/ production/deliverables/EURAKNOS-Policy-Brief.pdf (accessed on 10 October 2021).

46. Maynard, A.D. How to Succeed as an Academic on YouTube. Front. Commun. 2021, 5, 130. [CrossRef] 